\title{
COTARD SYNDROME IN HYPOACTIVE DELIRIUM - A CASE REPORT
}

\author{
Raimund Oberndorfer ${ }^{1,2}$, Christoph Schönauer ${ }^{1}$, Hans Eichbauer ${ }^{1}$, \\ Klaus Klaushofer ${ }^{1,3}$ \& Fabian Friedrich ${ }^{1,2}$ \\ ${ }^{1}$ First Medical Department, Hanusch-Hospital, Vienna, Austria \\ ${ }^{2}$ Department of Psychiatry and Psychotherapy, Division of Social Psychiatry, \\ Medical University of Vienna, Vienna, Austria \\ ${ }^{3}$ Ludwig Boltzmann Institute of Osteology, Hanusch-Hospital, Vienna, Austria
}

received: 17.10.2016;

revised: 27.2.2017;

accepted: 5.4 .2017

$* * * * *$

\section{INTRODUCTION}

Cotard syndrome (CS) is a rare condition centered around nihilistic delusions, ranging from the denial of body parts to that of the existence of ones own life or even the entire universe (Debruyne et al. 2011). Originally termed délire des négations by French neurologist Jules Cotard (1840-1889), CS has been primarily and predominantly described in the context of depression. Subsequently, it was also associated with other psychiatric (schizophrenia, bipolar disorder) as well as somatic conditions (Debruyne et al. 2011).

Delirium is an organic psychiatric syndrome that can be induced by various general medical causes, as well as by a range of substances and medication side effects. It is characterised by an acute onset, fluctuating course of symptoms, impaired level of consciousness and disturbance of cognition (e.g. disorientation, memory impairment, alteration in language, inattention). Supportive features include disturbance in sleep-wake cycle, perceptual disturbances (hallucinations or illusions), delusions, psychomotor disturbance (hypo- or hyper-activity), inappropriate behavior, and emotional lability (Inouye et al. 2014). Leading risk factors are dementia or cognitive, functional, or vision impairment, history of alcohol abuse, and advanced age ( $>70$ years) (Inouye et al. 2014). Delirium is associated with a poor long term outcome, i.e. an increased risk of death, institutionalisation, impairment and dementia (Witlox et al. 2010), as well as high healthcare costs (Inouye et al. 2014). Counterintuitively, a recent study suggested that even patients without a given risk factor experience adverse outcomes after delirium at least as often as do those with preexisting risk factors (Witlox et al. 2010). Despite high occurrence rates, particularly in its hypoactive form - with prevalence ranging from $6.25 \%$ in consultation-liason psychiatric services to $100 \%$ in intensive care units - delirium is often unrecognised (Peritogiannis et al. 2015). Furthermore, since hypoactive delirium and depression clinically overlap, patients may be misdiagnosed as depressed or fatigued, unless a careful assessment of symptom character as well as the context and course of disturbances is performed (Farrell \& Ganzini 1995, O'Sullivan et al. 2014, Spiller \& Keen 2006).

Here we describe a rare case of a woman without preexisting mental disorders that developed CS in the course of a protracted hypoactive delirium caused by multiple organ dysfunction following a humerus fracture.

\section{THE CASE}

A 76-year-old woman with several preexisting somatic diseases (arterial hypertension, coronary artery disease, atherosclerosis of the aorta, peripheral artery occlusive disease stage I of both lower extremities after stent-implantations, non-insulin dependent type 2 diabetes mellitus, hepatic steatosis, chronic obstructive pulmonary disease Gold III and osteoporosis), but no previous history of mental disorders, had injured herself in a fall at home. Four days later, she was admitted to the trauma department of a general hospital, where a left subcapital humerus fracture was diagnosed and treated conservatively. Initial laboratory tests showed uremia, electrolyte imbalance, acute renal insufficiency, elevated liver enzymes, and elevated inflammatory parameters (white blood count (WBC) $15.16 \mathrm{G} / \mathrm{l}$, C-reactive protein (CRP) $12.28 \mathrm{mg} / \mathrm{dl}$ ). An empiric antibiotic therapy with ampicillin/sulbactam $3 \mathrm{~g}$ three times daily for eight days, as well as furosemide and fluid replacement were started immediately.

In the course of the next two days, the patient deteriorated drastically, developing rhabdomyolysis with both acute kidney and liver failure and dramatically increasing inflammatory parameters. Neither chest Xray, urine, stool and blood cultures, hepatitis serology, nor abdominal sonography provided evidence of a focus of infection. However, after eight days of treatment, and upon transfer to the department of internal medicine, kidney function had normalised and liver enzymes had decreased. The patient was afebrile, but inflammatory parameters were still elevated (WBC $18.4 \mathrm{G} / \mathrm{l}$, CRP $16.6 \mathrm{mg} / \mathrm{dl}$, procalcitonin $0.17 \mathrm{ng} / \mathrm{ml}$ ). No psychiatric symptoms had been documented until then. 
Upon transfer, signs of disorientation and inattention, followed by a drastic deterioration of overall cognitive performance in the course of the next days, became evident. The patient presented in a hypoactive state of delirium - with fluctuating vigilance and attention deficits, reduced psychomotor activity, decreased speech, restricted affect and anxiety - but without obvious hallucinations or depressive symptoms. Her symptoms culminated in the delusions of lacking exactly two milliliters of blood, being dead (having died 'one hour and two minutes too early to be able to die a natural death') and being immortal - thus, meeting the criteria of CS.

A psychiatric consultation confirmed the diagnosis of hypoactive delirium with CS. An antipsychotic therapy with quetiapine (Seroquel) $50 \mathrm{mg}$ once daily was established. Finally, an empiric antibiotic therapy with piperacillin and tazobactam $4.5 \mathrm{~g}$ intravenously three times daily for seven days led to decreased inflammatory parameters (CRP $8.1 \mathrm{mg} / \mathrm{dl}$ ), and complete resolution of psychotic and delirious symptoms was evident within a week. After that, the patient showed no signs of cognitive impairment (mini-mental state examination: 30/30 points). Except for sleep disturbing nightmares of her delirious experiences lasting for several days, the patient had no depressive or other psychiatric symptoms for the rest of her hospital stay and was discharged home soon after.

\section{DISCUSSION}

Here we describe a case of hypoactive delirium presenting with Cotard syndrome caused by a traumatic bone injury followed by rhabdomyolysis with kidney and liver failure and an increased inflammatory reaction (multiple organ dysfunction syndrome) of unknown origin. Whereas delirium is a common condition with multiple known predisposing and aetiological factors, $\mathrm{CS}$ is extremely rare and risk factors are essentially unknown.

Concerning the aetiology of CS, neuroimaging findings suggest an important role for the fronto-temporoparietal circuitry (Kudlur et al. 2007) and insular cortex (Chatterjee \& Mitra 2015), but in most studies major changes in brain morphology were absent (Kudlur et al. 2007). A fluorodeoxyglucose positron emission tomography case study of a patient with CS identified hypometabolism in a bilateral frontoparietal cortical network (Charland-Verville et al. 2103). A disconnection between the sensory areas and the limbic system, leading to a complete lack of emotional contact with the world which would then be responsible for the patient's impression of being emotionally 'dead' was suggested (Ramachandran \& Blakeslee 1998). Several associations between structural (e.g. cerebral atrophy, dilated lateral ventricles) and functional brain changes, involving the frontal cortex and various other brain regions, were reported in neuroimaging studies of both CS and delirium. For delirium research, promising neuroimaging techni- ques such as MRI, SPECT and others (Alsop et al. 2006) exist. However, the evidence of results remain inconsistent and careful interpretation of the data is required. Two recent MRI studies could show neither a significant association between brain atrophy or whitematter hyperintensities (Cavallari et al. 2015), nor between global or voxel-wise cerebral blood flow, with the incidence and severity of postoperative delirium (Hshieh et al. 2016) in nondemented elderly patients. These findings contrast with previous assumptions about neuroradiological risk factors for delirium (Brown et al. 2015, Soiza et al. 2008). In another study, the correlations of blood oxygen levels between various brain regions in resting-state fMRI scans of patients both during the acute episode and after its resolution were examined (Choi et al. 2012). A disruption in reciprocity of the dorsolateral prefrontal cortex with the posterior cingulate cortex and a reversible reduction of functional connectivity of subcortical regions, including the mesencephalic tegmentum (which relays brainstem reticular activation), the midbrain nucleus basalis (the source of cholinergic innervation), and the midbrain ventral tegmental area (the source of dopaminergic innervation), was shown.

Taking into account the lack of preexisting cognitive impairment or mental disorder, the emergence of CS in our patient seems exceptional.

According to the largest currently available description of 100 cases of CS, depression was reported in $89 \%$ of subjects. Furthermore, not surprisingly, being the central characteristic of CS, the most common nihilistic delusions concerned the body $(86 \%)$ and existence $(69 \%)$, followed by anxiety (65\%) and guilt $(63 \%)$, hypochondriacal delusions (58\%) and delusions of immortality (55\%) (Debruyne et al. 2011). CS has been described in the context of other uncommon monothematic delusions as well. Amongst them are Capgras syndrome, the delusion that (often related) others have been replaced by (near) identical others - e.g. after traumatic brain injury or in schizophrenia (Debruyne et al. 2011) - or simultaneously with Koro, the genital retraction syndrome (Meagher et al. 2013), and Fregoli delusion, the delusional misidentification of familiar people disguised as others (Wolff \& McKenzie 1995). It is noteworthy, that despite a number of published case reports of CS, the nosological circumstance of delirium, and especially hypoactive delirium, is mentioned in practically none of them.

\section{CONCLUSIONS}

The case highlights the crucial importance of a multidisciplinary approach to timely diagnose and treat delirious states, especially in geriatric settings. In this case, the hypoactive state of delirium, which is often unrecognised and misdiagnosed, together with CS, might have impeded early diagnosis, if it were not for the multidisciplinary work. The latter allowed for an immediate beginning of treatment from the first day of 
transfer to our specialized geriatric unit. Inflammation, electrolyte imbalance and organ failure were successfully treated.

Additionally, a supportive antipsychotic therapy with quetiapine, which has shown good response rates and a low incidence of extrapyramidal side effects (Meagher et al. 2013), was established.

CS and delirium have major implications for patients. Already subtle signs of possible, especially hypoactive delirium should lead to a more thorough evaluation. To avoid severe, potentially lethal complications, the implementation of appropriate diagnostic, preventive and therapeutic measures is recommended (Inouye et al. 2014).

\section{Acknowledgements: None.}

\section{Conflict of interest: None to declare.}

\section{Contribution of individual authors:}

Oberndorfer Raimund: primary author, literature research, medical treatment;

Schönauer Christoph: contribution to literature research and writing the article, medical treatment;

Eichbauer Hans: contribution to research of internal medicine aspects and writing the article, medical treatment;

Klaushofer Klaus: contribution to writing the article, supervision of internal medicine aspects, medical treatment;

Fabian Friedrich: contribution to writing the article and literature research, supervision of psychiatric aspects, medical treatment.

\section{References}

1. Alsop DC, Fearing MA, Johnson K, Sperling R, Fong TG, Inouye SK: The Role of Neuroimaging in Elucidating Delirium Pathophysiology. J Gerontol 2006; 12:1287-1293.

2. Brown $\mathrm{CH}$, Faigle $R$, Klinker L, Bahouth M, Max L, LaFlam A, Neufeld KJ, Mandal K, Gottesman RF, Hogue $C W$ : The Association of Brain MRI Characteristics and Postoperative Delirium in Cardiac Surgery Patients. Clin Ther 2015; 37:2686-2699.

3. Cavallari M, Hshieh TT, Guttmann CRG, Ngo LH, Meier $D S$, Schmitt EM, Marcantonio ER, Jones RN, Kosar CM, Fong TG, Press D, Inouye SK, Alsop DC, on behalf of the SAGES Study Group: Brain atrophy and white matter hyperintensities are not significantly associated with incidence and severity of postoperative delirium in older persons without dementia. Neurobiol Aging 2015; 36:2122-2129.
4. Charland-Verville V, Bruno MA, Bahri MA, Demertzi A, Desseilles $M$, Chatelle $C$, et al.: Brain dead yet mind alive: A positron emission tomography case study of brain metabolism in Cotard's syndrome. Cortex 2013; 49:1997-1999.

5. Chatterjee SS \& Mitra S: "I Do Not Exist" - Cotard Syndrome in Insular Cortex Atrophy. Biol Psychiatry 2015; 77:e52-e53.

6. Choi SH, Lee H, Chung TS, Park KM, Jung YC, Kim SI, Kim JJ: Neural Network Functional Connectivity During and After an episode of Delirium. Am J Psychiatry 2012;169:498-507

7. Debruyne H, Portzky M, Peremans $K$, Audenaert K: Cotard's Syndrome. Mind Brain 2011,2:67-72.

8. Farrell $K$ \& Ganzini L: Misdiagnosing delirium as depression in medically ill elderly patients. Arch Intern Med 1995;155:2459-2464

9. Hshieh TT, Dai W, Cavallari M, Guttmann CR, Meier DS, Schmitt EM, Dickerson BC, Press DZ, Marcantonio ER, Jones RN, Gou YR, Travison TG, Fong TG, Ngo L, Inouye SK, Alsop DC; SAGES Study Group: Cerebral blood flow $M R I$ in the nondemented elderly is not predictive of postoperative delirium but is correlated with cognitive performance. J Cereb Blood Flow Metab. 2016 Jan 1:271678X16656014.

10. Inouye SK, Westendorp RG, Saczynski JS: Delirium in elderly people. Lancet 2014; 383(9920):911-922.

11. Kudlur NC, George S, Jaimon M: An overview of the neurological correlates of Cotard syndrome. Eur $J$ Psychiatry 2007; 21:99-116.

12. Meagher DJ, McLoughlin L, Leonard M, Hannon N, Dunne C, O'Regan N: What do we really know about the treatment of delirium with antipsychotics? Ten key issues for delirium pharmacotherapy. Am J Geriatr Psychiatry 2013; 21:1223-38.

13. O'Sullivan $R$, Inouye $S K$, Meagher D: Delirium and depression: Inter-relationship and overlap in elderly people. Lancet Psychiatry 2014; 1:303-311.

14. Peritogiannis V, Bolosi M, Lixouriotis C, Rizos DV: Recent Insights on Prevalence and Corelations of Hypoactive Delirium. Behav Neurol 2015; 2015:416792.

15. Ramachandran VS \& Blakeslee S: Phantoms in the brain: Probing the Mysteries of the Human Mind. New York: William Morrow; 1998:p. 119.

16. Soiza RL, Sharma V, Ferguson K, Shenkin SD, Seymour DG, MacLullich AMJ: Neuroimaging studies of delirium: A systematic review. J Psychosom Res 2008; 65:239-248

17. Spiller $J$ \&Keen J: Hypoactive delirium: assessing the extent of the problem for inpatient specialist palliative care. Palliative Medicine 2006; 20:17-23.

18. Witlox J, Eurelings LS, de Jonghe JF, Kalisvaart KJ, Eikelenboom P, van Gool WA: Delirium in elderly patients and the risk of postdischarge mortality, institutionalization, and dementia: a meta-analysis. JAMA 2010; 304:443-51.

19. Wolff $G$ \& McKenzie K: Capgras, Fregoli and Cotard's syndromes and Koro in folie à deux. Br J Psychiatry 1994; 165:842.

Correspondence:

Raimund Oberndorfer, $M D$

First Department of Internal Medicine (1. Medizinische Abteilung), Hanusch-Hospital

Heinrich-Collin-Straße 30, 1140 Vienna, Austria

E-mail: raimund.oberndorfer@gmail.com 